\title{
LA ORGANIZACIÓN RETÓRICA DEL MARCO REFERENCIAL EN TESIS DE TRABAJO SOCIAL ${ }^{1}$
}

Rhetorical organization of the reference framework in Social Work graduation theses

\author{
Mónica Tapia Ladino* \\ Gina Burdiles Fernández**
}

Resumen

Los estudios de géneros discursivos han prestado poca atención a las tesis o seminarios producidos para la obtención del grado de licenciatura. En este artículo se describe, desde el enfoque del genre analysis (Swales, 1990), la organización retórica del marco referencial de un conjunto de 30 tesis de pregrado elaboradas por estudiantes de la carrera de Trabajo Social de la UCSC. Se identifican cuatro movidas retóricas: teórico, conceptual, empírico y normativo. Se observa que cada una tiene propósitos diferentes sobre cuestiones teóricas, prácticas y legales relacionadas con el problema social que aborda cada tesis. Se advierte la presencia de algunos pasos retóricos recursivo y, al mismo tiempo, un fuerte compromiso de la investigación con la resolución de un problema social real.

Palabras clave: Géneros académicos, organización retórica, tesis, movida retórica, trabajo social.

Abstract

Studies of discourse genre have paid little attention to graduation theses produced by undergraduate students. This article describes, from a genre analysis approach (Swales, 1990), the rhetorical organization of the reference framework of a set of 30 undergraduate theses prepared by social work students at the UCSC. The study identifies four rhetorical moves: theoretical, conceptual, empirical, and normative. It is observed that each move has different purposes on theoretical, practical, and legal issues related to the social problem addressed by each thesis. The presence of recursive rhetorical steps is apparent, along with a strong commitment to solving real social problems through research.

Key words: Academic genres, rhetorical organization, thesis, rhetorical move, social work.

\section{ANTECEDENTES SOBRE ESCRITURA ACADÉMICA}

La presente investigación forma parte de una iniciativa mayor que tuvo como fin averiguar diversos aspectos del proceso de escritura en algunas disciplinas durante la etapa final de la formación académica en las áreas de Ciencias y Humanidades en dos Universidades del Consejo de Rectores de Chile. Como sabemos, en la universidad los estudiantes necesitan producir una diversidad de

\footnotetext{
${ }^{1}$ Resultado de investigación en el marco del Proyecto Fondecyt 1090151.
} 


\section{Mónica Tapia Ladino, Gina Burdiles Fernández}

textos orales y escritos para acreditar sus procesos de aprendizaje. Los exámenes y el desarrollo de diversos informes académicos (Harvey, 2005; Núñez y Espejo, 2005; Tapia y Burdiles, 2009) así como la lectura de manuales (Parodi, 2008a, 2008b) son, entre otros, los medios a través de los cuales los estudiantes universitarios demuestran el dominio de los contenidos y de las habilidades para las cuales son preparados durante su formación. Los tipos de escritos producidos en dichos contextos se han considerado como expresiones del discurso académico (DA), es decir, de aquel modo de comunicación típico que los usuarios del mundo universitario utilizan para vehicular los conocimientos estabilizados y aquellos que están en fase de producción (Venegas, 2007; Parodi, 2008c). El DA se ha entendido como un término amplio que incluye una variedad de productos orales y escritos: artículos, informes, tesis, defensas de tesis, entre muchos. En Chile, Harvey y Muñoz (2006) han señalado que la producción de escritos académicos es llevada adelante por los miembros de la universidad con el fin de establecer diferentes relaciones como co-construir, transmitir y difundir el conocimiento.

\section{MARCO TEÓRICO}

\subsection{Géneros académicos}

Una manera de acercarse a la particularidad de los discursos académicos ha sido mediante la aplicación del concepto de género discursivo sugerido por Bajtín (1985), término que se ha utilizado para referirse a aquellas formas de discurso que se han fijado por el uso y que se repiten con relativa estabilidad en las mismas situaciones comunicativas. Por esta razón, los hablantes reconocen los géneros, los distinguen por su forma y los asocian a situaciones comunicativas específicas. Los estudios sobre los géneros discursivos fueron posteriormente ampliados por Swales (1990) al proponer el concepto de comunidades discursivas para referirse a los grupos de personas que comparten un conjunto de prácticas comunicativas con características léxicas, textuales y discursivas con las que se alcanzan las metas persuasivas del grupo. Desde esta perspectiva, el mundo universitario es una comunidad que presenta modos de comunicación característicos, cuyos textos presentan rasgos particulares que los estudiantes requieren aprender para integrarse progresivamente al grupo y así ser parte de la comunidad académica.

Diversas universidades del hemisferio norte han llevado adelante iniciativas orientadas a desarrollar en sus estudiantes las habilidades necesarias para producir las distintas modalidades del discurso académico. Estas iniciativas se han basado en el supuesto de que las áreas epistemológicas de formación determinan las formas que adquieren dichos escritos (Bazerman, 1988; Berkenkotter y Huckin, 1995); es decir, las áreas disciplinares de formación determinan las características de los textos y, por tanto, cuando se estudia un área del saber es necesario aprender tanto los contenidos como los modos particulares en que se vehicula dicho conocimiento. 


\section{La organización retórica del marco referencial en tesis de Trabajo Social}

En los últimos años se ha producido un creciente interés por abordar el discurso académico en sus diferentes manifestaciones, tanto por su pertinencia para la formación académica como para la profesional. Tal interés se remonta a los estudios realizados desde la lingüística aplicada en el ámbito anglosajón (Bhatia, 1993, 2002a, 2002b, 2004). Estas investigaciones presentaban una orientación pedagógica, pues buscaban proponer nuevos enfoques didácticos para facilitar la enseñanza del inglés con propósitos específicos y académicos (Swales, 1990, 2001; Flowerdew y Peacock, 2001; Flowerdew, 2002). Tal preocupación encontró eco en nuestro país, donde ha surgido un especial interés por el estudio del discurso académico. Destacan, en este contexto, las investigaciones de Tapia, Burdiles y Arancibia (2003), Núñez y Espejo (2005), Parodi (2005, 2007, 2008a, 2010), Venegas (2007), quienes se han dedicado a estudiar los textos académicos y profesionales especialmente adoptando el enfoque de los géneros discursivos (Swales, 1990; Bhatia, 1993, 2002a, 2004). Los hallazgos han revelado que los textos solicitados en la formación universitaria están orientados a demostrar el saber y el hacer, es decir, los estudiantes requieren escribir para acreditar el dominio de contenidos, así como demostrar capacidades para hacer aquellas actividades que les serán exigidas en el ámbito profesional - laboral.

Estudios sobre los escritos exigidos durante la formación universitaria revelan que son los informes académicos los más solicitados (Harvey, 2005; Tapia Ladino y Burdiles, 2009). A pesar del creciente interés por el estudio de los textos académicos, son aún insuficientes los trabajos que abordan los escritos mayores como las tesis de grado o seminarios de investigación. Conocer con mayor profundidad dichos textos, nos permitiría enfrentar de mejor manera el proceso de enseñanza.

\subsection{La tesis, un género discursivo}

Como ya se ha señalado, la educación universitaria, de manera permanente, enfrenta a los estudiantes a géneros escritos que pueden ser desconocidos para ellos, por lo que comúnmente manifiestan dificultades ante tareas de escritura académica de gran envergadura como la tesis.

Nuestras investigaciones revelan que enfrentar la escritura de una tesis es una actividad altamente demandante tanto para los estudiantes como para los docentes que realizan la tarea de guía (Marinkovich y Velásquez, 2010; Tapia Ladino y Marinkovich, 2011). Los resultados revelan que las representaciones sociales que surgen de la experiencia de escribir y guiar una tesis varían entre estudiantes y académicos. Tales diferencias producen tensiones al interior de la comunidad, pues los estudiantes perciben la escritura de la tesis como una tarea compleja para la que no se sienten preparados $\mathrm{y}$, los académicos, por su parte, tienden a asumir el desarrollo de dichos escritos como una etapa en la formación de sus estudiantes, es decir, una fase para ingresar a los estudios de posgrado.

La tesis es un trabajo de graduación, de pregrado o postgrado, que informa una investigación sustentada en conocimientos y razonamientos teóricos, desarrollada en base a métodos y técnicas científicos. Según Marimón y Santamaría 


\section{Mónica Tapia Ladino, Gina Burdiles Fernández}

(2007), el propósito de una tesis es comunicar el resultado de investigaciones científicas $\mathrm{y}$, en este sentido, se asemejaría al ensayo científico, al artículo científico, al informe técnico-científico y a la monografía de investigación.

Mucho de lo que se ha publicado sobre las tesis se ha hecho en términos de orientaciones prescriptivas que regulan el formato de su escritura (Day, 1996; Eco, 1997; Bolker, 1998; Calderón de la Barca \& Bono, 2003; Aleskerov, 2009).

En las últimas dos décadas se han incrementado los estudios lingüísticos que analizan y describen el género tesis, sobre todo las de postgrado. Entre estos estudios destaca el de Paltridge y Starfield (2007), quienes describen el patrón estructural típico de una tesis conformada por una introducción, revisión de la literatura, materiales y métodos, resultados, discusión y conclusión. En relación con las tesis de pregrado - que han sido menos estudiadas - destacan, a nivel regional, los estudios de Marinkovich y Velásquez (2010), Tapia Ladino y Marinkovich (2011) y Marinkovich y Salazar (2011). Estas investigaciones han dado cuenta de las representaciones sociales de los actores del proceso educativo en torno a la escritura de las tesis de pregrado en diversas carreras universitarias.

La creciente valoración del análisis del género como herramienta útil para dar cuenta de sus patrones organizacionales, ha dado pie en los últimos treinta años a numerosas investigaciones, principalmente en lengua inglesa. En ese contexto se ha desarrollado un número importante de investigaciones centradas en secciones específicas de las tesis de diversas disciplinas. Algunos estudios han examinado el subgénero introducción en trabajos de grado, como la tesis doctoral de Química, Ecología y Biodiversidad (Bunton, 2002); la tesis de magíster en Biología, Filosofía y Lingüística (Samraj, 2008) y las introducciones en tesis doctorales de Química y Lingüística (Jara, 2009). Otra sección que ha concitado el interés de estudios de este tipo es la conclusión, la que ha sido observada en tesis doctorales de Arte, Educación, Ciencias Sociales, Arquitectura, Ingeniería, Ciencias, Medicina, Odontología, Economía y Manejo Ambiental (Bunton, 2005). Los apartados de revisión de la literatura o marco teórico no han sido objeto de investigación lingüística.

Otros trabajos han abordado el género en toda su extensión. Tal es el caso del estudio de las tesis doctorales de Ciencia, Ingeniería, Arte, Educación y Ciencias Sociales (Bunton, 1998). En esta misma línea, y a partir de la observación de los patrones de organización de las tesis, se han esbozado clasificaciones atendiendo a la mayor o menor complejidad de su estructura interna (Dudley-Evans 1986). Todos estos estudios apuntan a señalar que en la organización retórica de un mismo género existen variaciones que guardan relación con variaciones disciplinares. En términos generales, además, valoran este tipo de hallazgos por la importancia que tienen para la enseñanza de la escritura académica y científica.

\subsection{El análisis del género}

El análisis del género (genre analysis) es un método que estudia los rasgos retórico-discursivos de textos que constituyen un género, en tanto 
comparten determinados patrones comunicativos y son reconocidos como tal por los miembros de una comunidad discursiva. Fue propuesto por Swales $(1981,1990,2004)$ para describir la organización de una sección del artículo de investigación científica publicado en inglés, con miras a implementar mejoras en los procesos de enseñanza de inglés con propósitos específicos.

El análisis del género consiste en identificar el conjunto de movidas (moves) o unidades retóricas asociadas a propósitos comunicativos específicos, que configuran la estructura - más o menos convencionalizada - que caracteriza a un género. Los propósitos comunicativos constituyen el criterio fundamental para definir un género, para determinar sus alcances, para configurar su estructura esquemática y para guiar las elecciones de su contenido y estilo (Swales, 1990). Bhatia (1993) subraya que el conjunto de estos propósitos —que se entienden compartidos por la comunidad discursiva - configura al género y le da una estructura interna. La configuración particular que es objeto de este estudio corresponde a la organización retórica, la que se entiende como "la representación de la estructura funcional de un género, a partir de la sistematización de sus unidades y subunidades retórico-discursivas" (Burdiles, 2011: 28). La unidad discursiva básica para desarrollar este análisis es la movida, definida por Swales (2004) como una unidad discursiva o retórica que realiza una función comunicativa coherente, en un discurso oral o escrito. Este enfoque, según Kanoksilapatham (2005) apunta a hacer una representación del género en términos de una organización jerárquica, formada por distintos niveles, las cuales pueden ser subdivididos en movidas y cada una de ellos, a su vez, se puede dividir en pasos. Dicho de otro modo, las movidas y los pasos responden a propósitos comunicativos que presentan diferentes niveles de abstracción.

\section{Metodología DE ANÁLISIS}

La presente investigación corresponde a un diseño descriptivo que especificó los rasgos de un fenómeno y sus tendencias, a saber, las propiedades de la organización retórica de un apartado de las tesis de pregrado en trabajo social. El estudio está basado en corpus, pues en este caso se busca estudiar la lengua a través de la observación de ejemplos reales de su uso en contextos académicos.

En conformidad con el objetivo, el marco metodológico de esta investigación fue el análisis del género a partir de las movidas retóricas (Swales, 1981, 1990, 2004). Para identificarlas se siguieron las orientaciones de Biber, Connor y Upton (2007), en lo referido a los pasos analíticos para lograr una descripción de estructura de discurso basada en corpus; asimismo, se siguió en gran medida el trayecto metodológico descrito por Parodi $(2008 b, 2008 \mathrm{c})$ en un estudio que da cuenta de la organización retórica de un género académico. En consecuencia, el análisis consideró primero el desarrollo de un marco analítico funcional y, posteriormente, su aplicación a los textos, en busca de las unidades del discurso (movidas). Se 


\section{Mónica Tapia Ladino, Gina Burdiles Fernández}

empleó una metodología que se sitúa entre los enfoques denominados descendentes $\mathrm{y}$ ascendentes, en tanto se complementan los enfoques deductivo e inductivo.

\subsection{El corpus}

El corpus está constituido por la totalidad de los marcos de referencia de las tesis de Trabajo Social de la UCSC producidas entre los años 2006 y 2009, esto es, 30 tesis o seminarios de titulación.

\subsection{Procedimientos}

En primer término, se recolectaron las tesis que se encontraban almacenadas en la estantería abierta de la Biblioteca Central de la mencionada casa de estudios. Luego, a fin de disponer de archivos digitales, se escaneó la totalidad de las tesis y se transformaron a archivos de texto. Finalmente, se eliminaron los títulos y subtítulos consignados originalmente, tales como "marco teórico", "marco conceptual, "marco referencial", etc. El objetivo de esta edición de los originales fue evitar que las pistas textuales de los subtítulos influyeran en la definición de los segmentos retóricos y sus propósitos comunicativos.

\subsection{Análisis de los datos}

Para realizar el análisis de los datos se siguieron los pasos que se sintetizan a continuación:

1. Aproximación general al corpus mediante una revisión minuciosa de las tesis completas.

2. Identificación en cada texto del gran segmento textual-discursivo en el que se sustenta la investigación objeto de las tesis.

3. Ambas investigadoras analizaron conjuntamente un microcorpus, en el que se identificó un grupo de unidades discursivas que parecían responder a distintos propósitos comunicativos.

4. Elaboración de una primera grilla de análisis a partir de las unidades retóricas.

5. Aplicación individual de la grilla a otro microcorpus.

6. Análisis conjunto de los resultados de la aplicación de la grilla, a fin de ecualizar y reordenar las unidades y subunidades halladas.

7. Diseño de la grilla definitiva.

8. Análisis completo del corpus.

9. Cuantificación de las ocurrencias de las unidades y subunidades retóricas.

10. Análisis de los datos cuantitativos, consistente en calcular las frecuencias relativas.

\section{Resultados}

Con el fin de obtener los resultados, realizamos cálculos simples de frecuencia de las unidades retóricas halladas. En la Tabla 1, presentamos la totalidad de movidas y pasos identificados en el marco de referencia de las 30 tesis de Trabajo Social revisadas. Los datos se organizan según tres tipos de unidades retóricas: macromovida, movida y pasos. Esta distinción corresponde a la observación de unidades retóricas de distinto nivel de abstracción en concordancia con el modelo de análisis. Los pasos permiten la realización de las 
movidas $\mathrm{y}$, a su vez, las movidas corresponden a un macropropósito comunicativo que se concreta en la macromovida (Parodi, 2008b, 2008c). Dicha categoría permite dar cuenta de macroniveles de análisis que facilitan una mejor descripción de unidades discursivas extensas.

TABLA 1. UNIDADES RETÓRICAS DEL MARCO REFERENCIAL EN TESIS DE TRABAJO SOCIAL

\begin{tabular}{|l|l|l|l|}
\hline \multicolumn{2}{|l|}{ Unidades retóricas } & \multicolumn{2}{l|}{ Cantidad } \\
\hline \multirow{4}{*}{$\begin{array}{l}\text { Macromovida: } \\
\text { Presentar respaldo } \\
\text { de la investigación }\end{array}$} & Movida 1: Despliegue de enfoques teóricos & 5 pasos \\
\cline { 3 - 4 } & & Movida 2: Declaración de un contexto normativo & 5 pasos \\
\cline { 2 - 4 } Total & 1 & Movida 3: Reseña de estudios previos & 4 pasos \\
\cline { 2 - 4 } & 1 & 4 & 5 pasos \\
\hline
\end{tabular}

Como se aprecia en la Tabla 1, para la macromovida presentar respaldo de la investigación identificamos cuatro movidas constituidas, a su vez, por entre 4 ó 5 pasos que se comentarán más adelante.

En relación con las cuatro movidas retóricas individualizadas, llama la atención que a la postre sus propósitos comunicativos presentaron correspondencia de uno a uno con los apartados subtitulados de los textos originales. Debe recordarse que el análisis se hizo sobre un corpus carente de subtítulos, pues se quiso evitar que las etiquetas determinaran la identidad de los propósitos retóricos identificados. Tal procedimiento explica el hecho de que realizáramos la distinción entre macromovida, movidas y pasos, pues aunque todos hacen referencia al propósito comunicativo de diferentes segmentos discursivos, nos interesaba dar cuenta de dichos fines en los diferentes niveles del escrito.

El análisis general del marco referencial de las tesis revela que en la carrera de Trabajo Social se delimitan claramente los temas, distinguiendo entre los aspectos teóricos, normativos, empíricos y las elecciones conceptuales a partir de las cuales se aborda el tema de la investigación.

En el gráfico 1, se presenta el detalle de las unidades retóricas que realizan la primera movida. El análisis reveló que el objetivo de esta movida era realizar el despliegue de los enfoques teóricos que sustentan la tesis. 
GrÁFico 1. Distribución de pasos en la Movida 1: Despliegue De ENFOQUES TEÓRICOS

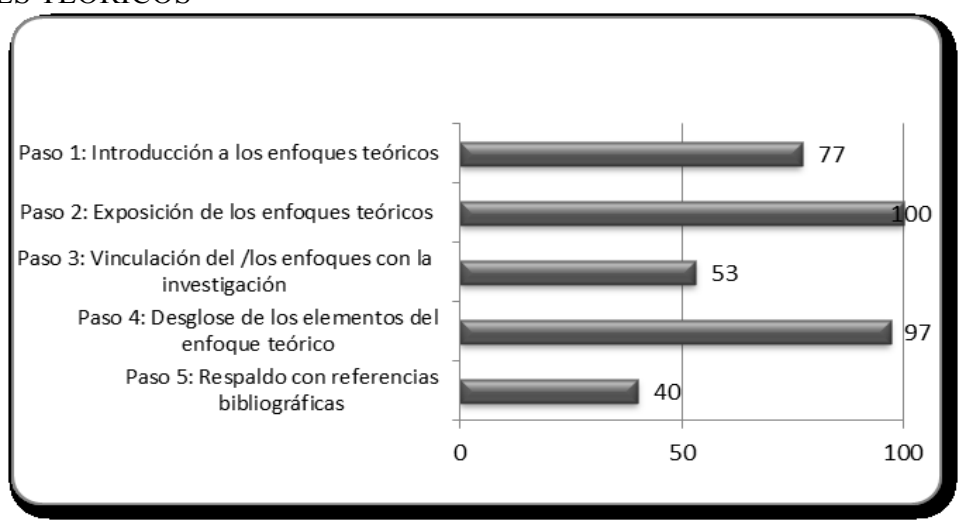

Al revisar el gráfico 1, advertimos que los pasos más frecuentes de la presentación de los enfoques teóricos corresponden al que aborda la exposición de los enfoques teóricos seguido por el paso desglose de los elementos que conformaban tal o tales teorías. Con menor frecuencia aparece el paso 1 que consiste en anunciar o de algún modo introducir los enfoques teóricos. Los paso 3 y 5 , es decir, vinculación de los enfoques con la investigación y el respaldo con referencias bibliográficas para presentar las teorías fueron los pasos que ocurrieron con menor frecuencia.

La distribución de frecuencia revela que los pasos 2 y 4 responden a propósitos comunicativos obligatorios que sirven para anunciar y dar cuenta de la teoría y sus componentes. Los demás pasos resultan menos obligatorios dados sus menores porcentajes. Por otro lado, llama la atención que el uso de referencias bibliográficas presente una baja ocurrencia, pues es esperable que los antecedentes teóricos sean respaldados con sus fuentes. Sobre este dato es necesario agregar que durante el análisis se observó que en la mayoría de los casos era dificultoso distinguir entre el discurso de los investigadores y el de las fuentes. Es decir, son pocas las ocasiones en las que se realiza la referencia a fuentes usando los sistemas de citación convencional. El análisis revela que en la movida 1 hay más bien una tendencia a la realización de resúmenes de las ideas de las fuentes y una baja utilización de sistemas de referencia para integrar el discurso de otros.

La revisión de las siguientes movidas 2, 3 y 4 dejará en evidencia que el uso de referencias bibliográficas para respaldar los temas de la investigación es un propósito que reaparece. Sin embargo, es en la movida 1 donde presenta la frecuencia más baja.

En el gráfico 2 presentamos la distribución de pasos identificados en la movida Referencias Normativas. Los análisis indican que este subapartado tenía la función de desplegar los antecedentes normativos en los que se enmarcaba el problema abordado por la tesis. 
GrÁFICO 2. Distribución de PASOS DE LA MOVIDA 2: DEClaración DE UN CONTEXTO NORMATIVO

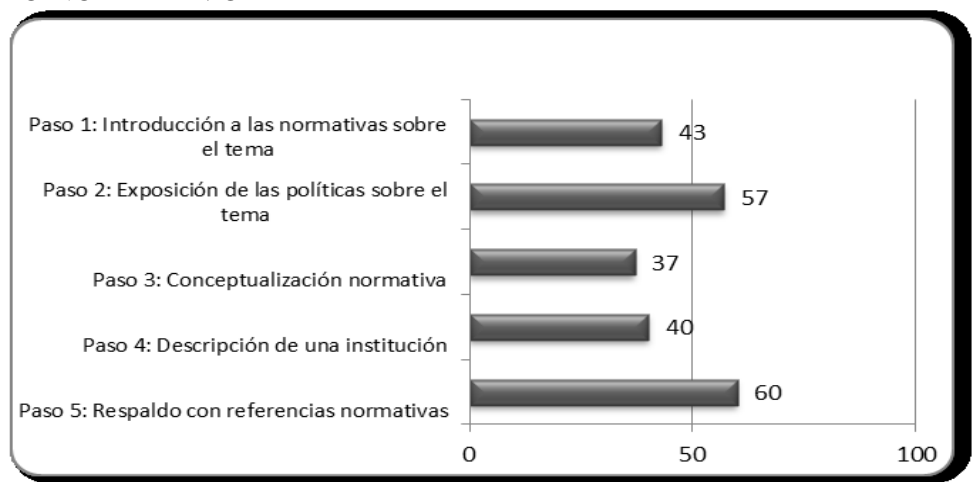

Como se aprecia en el Gráfico 2, los pasos que constituyen la movida 2 presentan, en general, una menor frecuencia en comparación con los de la movida 1 , la que oscila entre el $37 \%$ y el $60 \%$. Al igual que en la movida 1 , esta comienza con el paso Introducción a las normativas sobre el tema en el que se contextualiza la investigación de la tesis. Los pasos más frecuentes se relacionan con la revisión de la normativa nacional e internacional (paso 2) y con el uso de referencias para explicar las leyes (paso 5).

La revisión de los pasos de la movida 2 revela que se trata de una parte de la tesis que tiene como fin informar sobre todos los aspectos legales y normativos que circunscriben el tema de investigación. Por lo tanto, los autores requieren aquí mencionar explícitamente las leyes que regulan el fenómeno. En el caso de existir poca jurisprudencia nacional o en el caso de que el tema sea de interés internacional, los investigadores hacen mención a normativas externas provenientes de tratados o convenciones internacionales.

Los antecedentes se configuran en dos escenarios posibles: uno para evidenciar que la importancia del tema amerita el estudio o bien que existe escasa normativa, por lo que se necesita más regulación para enfrentar la resolución del problema de investigación.

En el gráfico 3, presentamos los pasos que realiza la movida 3 la cual hace referencia a los estudios previos realizados en torno al tema de investigación de la tesis. Esta movida tiene como objetivo proveer antecedentes empíricos destinados a evidenciar el interés que ha provocado el tema en otros investigadores sobre el tema. 


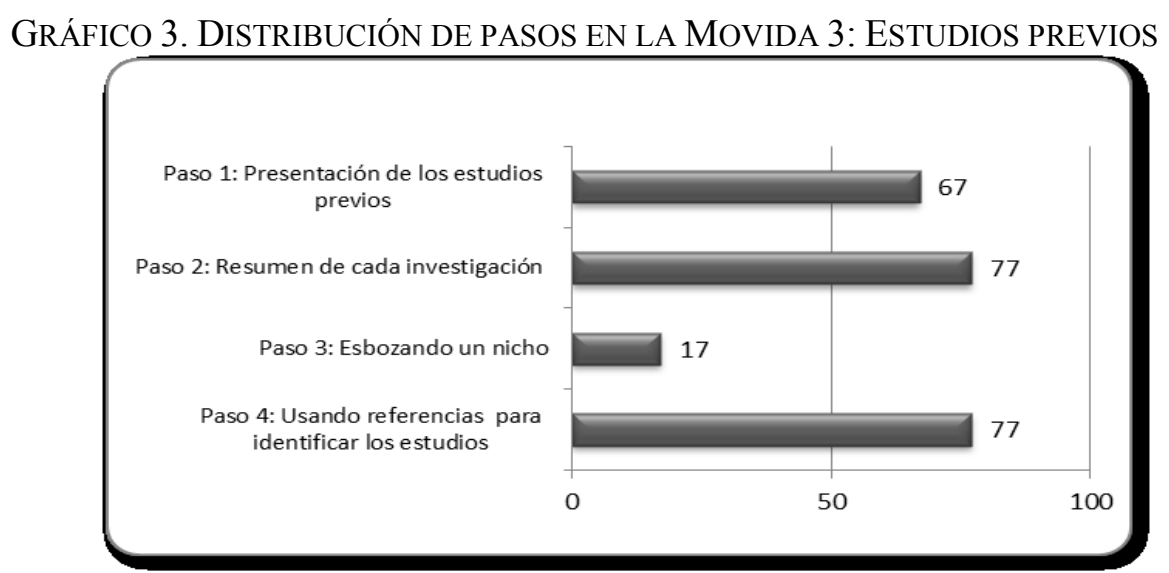

El gráfico 3 informa que la movida 3 se realiza a través de 4 pasos. Al igual que en las movidas anteriores, esta comienza con un anuncio o presentación de los estudios previos. Los pasos más recurrentes fueron resumen de cada investigación (77\%) y respaldo con referencias bibliográficas (77\%) para presentar las investigaciones. En esta movida retórica, normalmente los autores hacen mención general sobre los estudios empíricos que se han realizado sobre el tema de investigación y luego cada investigación es presentada de manera sintética en una secuencia en la que no se advierte alternancia entre el discurso propio y el ajeno. En esta unidad retórica, en general, se provee de un reporte breve mediante la elaboración de párrafos con las ideas principales sobre los aspectos más relevantes de la investigación: teoría en la que se sustentan, método y diseño de la investigación, rasgos de los participantes y principales resultados.

Es necesario comentar que en esta movida se observó una yuxtaposición de tales resúmenes y llama la atención que no se realicen comentarios a los estudios y no se establecen relaciones entre ellos. En estos casos, el sistema de referencia bibliográfica solo sirve para presentar la fuente.

En el gráfico 4 se presenta la movida relacionada con las opciones conceptuales de la investigación. Esta unidad retórica se propone como un glosario en el que se presentan las definiciones de los conceptos que más adelante en la tesis se funcionalizan y operacionalizan en la metodología y los resultados de la investigación. 


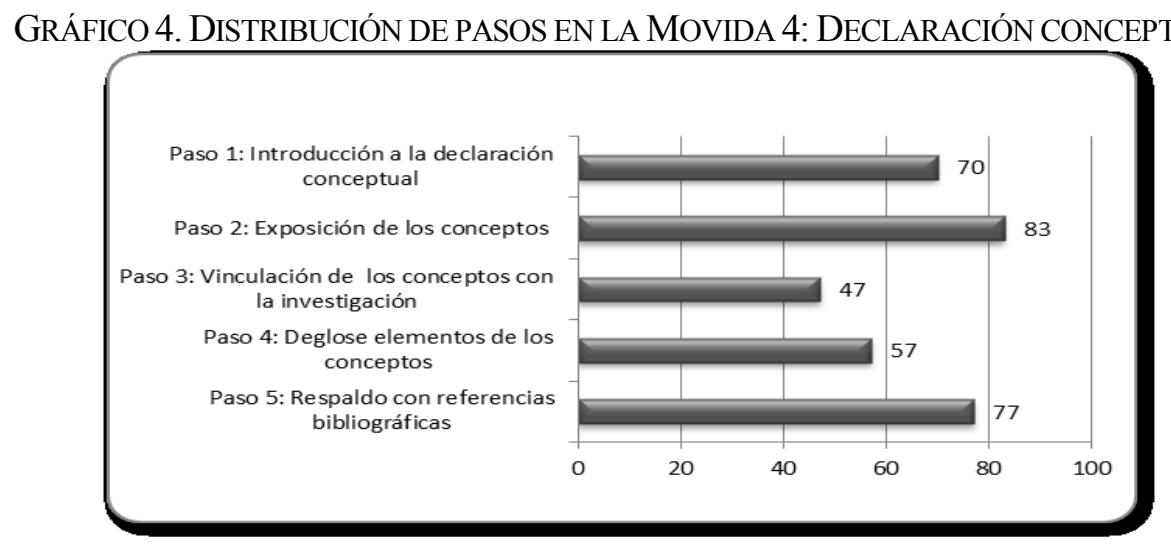

Como se observa en el gráfico 4, al igual que en las movidas anteriores, el apartado comienza con una presentación del tema $(70 \%)$. Los pasos más frecuentes fueron exposición de los conceptos (83\%) y respaldo con referencias bibliográficas (77\%). El paso 3 sobre vinculación de los conceptos con la investigación consiste en que los autores justifican la elección conceptual a la luz de la investigación. Sobre el paso 4 desglose de los elementos de los conceptos, se trata de la descomposición de los temas definidos. Por ejemplo, si se aborda el tema de la violencia intrafamiliar, se definen uno a uno los tipos de violencia de esta naturaleza.

En términos generales, la movida 4 se caracteriza por exponer los conceptos como si se tratara de un diccionario ad hoc a los propósitos de la investigación. En él se señala cada noción y se la define operativamente como respondiendo a la pregunta ¿Qué entendemos por este concepto en esta investigación? Cada concepto, a menudo, hace referencia a las fuentes bibliográficas.

\section{DISCUSIÓN}

La herramienta de análisis mediante la identificación de las unidades retóricas jerarquizadas en niveles resultó de suma utilidad, pues permitió capturar la organización comunicativa de una parte de la tesis de Trabajo Social destinada a comunicar los distintos respaldos de la investigación. El análisis hace evidente que la investigación enfrenta el tema desde cuatro ángulos que van desde lo abstracto o teórico a lo procedimental. La manera de organizar los apoyos concuerda con los resultados de una investigación anterior en la que describimos las representaciones sociales sobre la tesis en estudiantes y profesores de esta carrera. Los hallazgos revelan que la producción de la tesis está fuertemente determinada por el medio profesional (Tapia Ladino y Marinkovich, 2011). En este contexto, la elaboración de la tesis evidencia el esfuerzo formativo orientado a elaborar un tipo de texto que esté al servicio de la sociedad.

Sobre la organización retórica de los pasos, se advierte la tendencia a la presentación y desglose de temas en cada una de las movidas. El análisis revela que existe un conjunto de pasos que se repiten en las movidas, es decir, presentan un 


\section{Mónica Tapia Ladino, Gina Burdiles Fernández}

comportamiento cíclico (Swales, 1990). Por ejemplo, la Introducción de los temas (teorías, normas, conceptos o estudios empíricos) y la consecuente exposición de dichos temas son pasos que se repiten a lo largo de las cuatro movidas. En términos de Sabaj (2011) se trata de unidades retóricas multiseccionales que aparecen en diferentes apartados del marco referencial y lo hacen en un mismo orden. Por su parte, el paso respaldo con referencias bibliográficas es una unidad retórica que, al mismo tiempo, es cíclica y multiseccional. Se presenta en las cuatro movidas y se repite al interior de cada una de ellas. Sin embargo, el poco uso de sistemas de referenciación evidencia que los respaldos en los que se apoya la investigación parecen ser concebidos como telón de fondo, lo que se expresa en una escasa discusión y controversia de ideas. Los investigadores hacen mención de cada uno de los temas y lo hacen con el fin de demostrar a su comunidad que están al tanto de ellas. Desde el punto de vista de lo señalado por Padilla (2010), se trata de textos que argumentan académicamente privilegiando el discurso demostrativo, es decir, articulando el marco teórico, con las hipótesis, los datos y conclusiones. Sin embargo, la autora señala que la argumentación académica también exige otros tipos de razonamientos como el dialéctico que se abre a la consideración de otros puntos de vista y que se asume como discurso provisional y perfectible (3). Los hallazgos de la autora demuestran que los aprendices universitarios avanzan durante su formación desde la argumentación demostrativa a la argumentativa.

Llamó la atención la existencia de la movida 2 que explícitamente hace mención al contexto normativo o legal del tema de investigación, pues revela el fuerte nexo que existe entre la profesión, los temas que son objeto de preocupación y el mundo real en que se circunscribe la indagación. Se trata de problemas altamente comprometidos con la sociedad lo que, sin duda, se explica por el carácter de las disciplinas que sustentan la formación de la carrera de Trabajo Social.

En relación con la movida sobre los estudios empíricos, tradicionalmente en el mundo científico la revisión de dichos estudios ha permitido identificar los nichos que serán ocupados por una investigación en curso. El análisis de las tesis de Trabajo Social evidencia que la identificación de un vacío en esta área funciona como una estrategia que da cuenta de la apropiación de un discurso más académico que profesional. Sin embargo, la baja ocurrencia de este paso en las tesis revisadas indica que las decisiones investigativas se realizan más bien por razones teóricas o legales, es decir, es la importancia teórico-social-legal la que define el abordaje de un tema de investigación en esta disciplina.

La inclusión de un apartado con elecciones conceptuales, como se observó en la movida 4, revela la necesidad de operacionalizar el marco teórico, normativo y empírico en conceptos susceptibles de hacer funcionar en la realidad. Como se ha venido señalando, el hecho de que las tesis de Trabajo Social estén orientadas a resolver un problema real, exige que los términos utilizados y aplicados estén libres de controversia, razón por la que denominamos a esta macromovida como elección conceptual. 
La revisión general de las unidades retóricas y comunicativas del Marco Referencial de las tesis de Trabajo Social revela el alto compromiso de esta disciplina con la sociedad. En esta carrera, la investigación es asumida como una tarea orientada a la resolución de un problema social. Tal objetivo parece determinar la elaboración de Marco Referencial para la investigación que se hace cargo de los aspectos teóricos, empíricos, normativos y procedimentales de la investigación. El análisis de las unidades retóricas que constituyen esta parte de las tesis de Trabajo social puede constituir un insumo para abordar los procesos de enseñanza del género tesis.

Universidad Católica de la Santísima*
Facultad de Educación
Alonso de Ribera 2850, Paicavíaltura 3000. Concepción (Chile)
mtapia@ucsc.cl
Universidad Católica de la Santísima**
Facultad de Educación
Alonso de Ribera 2850, Paicavíaltura 3000. Concepción, Chile.
gburdiles@ucsc.cl

BIBLIOGRAFÍA

Aleskerov, Fouad. How to prepare and write $\mathrm{PhD}$ and MS thesis. En Automation and Remote Control, 70 (11). 2009:177-188.

Bazerman, Charles. Shaping written knowledge: the genre and activity of experimental article in science. Madison: University of Wisconsin Press. 1988.

Bajtín, Mijail. Estética de la creación verbal. México: Siglo XXI. 1985.

Bhatia, Vijay. "A Generic View of Academic Discourse". John Flowerdew (Ed.). Academic Discourse. Cambridge: Cambridge University Press. 2002a:21-39.

------ "Applied genre analysis: Analytical advances and pedagogical procedures". En Genre in the classroom: Multiple perspectives A Johns (ed), Lawrence Erlbaum Associates, Publishers, London, United Kingdom. 2002b:279-283.

------ Analysing Genre. Language use in professional settings. London: Longman. 1993.

Bhatia, Vijay. Worlds of written discourse. A genre-based view. New York: Continuum. 2004.

Berkenkotter, Carol; Huckin, Thomas. Genre knowledge in disciplinary communication. New York: Lawewnce Erlbaum Associates. 1995.

Biber, Douglas; Connor, Ulla; Upton, Thomas. Discourse on the Move: Using Corpus Analysis to Describe Discourse Structure. Amsterdam: Benjamins. 2007.

Bolker, Joan. Writing Your Thesis in Fifteen Minutes a Day: A Guide to Starting, Revising, and Finishing Your Thesis. New York: H. Holt. 1998.

Bunton, David. "The structure of $\mathrm{PhD}$ conclusion chapters". Journal of English for Academic Purposes, 4. 2005:207-224. 


\section{Mónica Tapia Ladino, Gina Burdiles Fernández}

----- "Generic moves in Ph.D. thesis introductions". John Flowerdew (Ed.), Academic discourse. London: Longman. 2002:57-75.

------ Linguistic and textual problems in $\mathrm{PhD}$ and M.Phil Theses: an analysis of genre moves and metatext. Tesis Doctoral, Universidad de Hong-Kong, Hong-Kong, China. 1998.

Burdiles, Gina. Descripción de la organización retórica del género Caso Clínico de la medicina a partir del Corpus CCM-2009. Tesis doctoral, Pontificia Universidad Católica de Valparaíso, Valparaíso, Chile. 2011.

Calderón de la Barca, Laura; Bono, María. Manual para la elaboración de tesis de Licenciatura. Número X, Seminario de Investigación Jurídica, México, D. F. 2003.

Day Robert. Cómo escribir y publicar trabajos cientificos. Washington: OPS/OMS. 1996.

Dudley-Evans, Tony. "Genre analysis: an investigation of the introduction and discussion sections of MSc dissertations". Malcom. Coulthard (Ed.). Talking about text. Birmingham:University of Birmingham. 1986.

Eco, Humberto, Cómo se hace una tesis. Técnicas y procedimientos de investigación, estudio y escritura. Barcelona: Gedisa. 1997.

Flowerdew, John; Peacock, Matthew. "Issues in EAP: A preliminary perspective". En John Flowerdew; Mattew Peacock (Eds.). Research Perspectivas on English for Academic Purposes. Cambridge: Cambridge University Press. 2001:315-359.

Flowerdew, John. (Ed.). Academic discourse. Cambridge: Cambridge University Press. 2002.

Harvey, Ana María. "La evaluación en el discurso de informes escritos por estudiantes universitarios chilenos". Mauricio Pilleux (Ed.). Contextos del discurso. Valdivia: Facultad de Filosofia y Humanidades. Universidad Austral de Chile. 2005:215-228.

Harvey, Ana María; Muñoz, Daniel. "El género informe y sus representaciones en el discurso de los académicos". Revista de Estudios Filológicos, 41. 2006:95-114.

Jara, Iván. Las introducciones en Tesis Doctorales de Química y Lingüistica: un estudio exploratorio desde el análisis de género. Tesis doctoral, Pontificia Universidad Católica de Valparaíso, Valparaíso, Chile. 2009.

Kanoksilapatham, Budsaba. "Rhetorical structure of biochemistry research articles". English for Specific Purposes, 24. 2005:269-292.

Marimón, Carmen; Santamaría, Isabel. "Los géneros y las lenguas de especialidad (II): el contexto científico-técnico". Enrique Alcaraz, José Martínez y Francisco Yus (Eds.). Las lenguas profesionales y académicas. Barcelona, Ariel. 2007:127-140.

Marinkovich, Juanita; Velásquez, Marisol. 'La representación social de la escritura y los géneros discursivos en un programa de licenciatura: una aproximación a la alfabetización académica". Giovanni Parodi (Editor). Alfabetización académica y profesional en el siglo XXI: leer y escribir desde las disciplinas. Santiago de 
Chile: Academia Chilena de la Lengua. Editorial Planeta. 2010:127-152.

Marinkovich, Juana; Salazar, Juan. "Representaciones sociales acerca del proceso de Escritura Académica: el caso de la tesis en una Licenciatura en Historia". Estudios Pedagógicos XXXVII, No 1. 2011:85-104.

Núñez, Paulina; Espejo, Carolina. "Estudio exploratorio acerca de la conceptualización del informe escrito en el ámbito académico". In Ana María Harvey (Comp.). En torno al Discurso. Contribuciones de América Latina. Santiago de Chile: Ediciones Pontificia Universidad Católica. 2005:135-148.

Padilla, Constanza; Douglas, Silvina; López, Esther. "La comunicación académica como construcción argumentativa: perspectivas de lectura y de escritura de estudiantes universitarios". XII Congreso de la Sociedad Argentina de Lingüistica (SAL). Panel de la cátedra UNESCO: "La argumentación y su enseñanza”. S, Ortega de Hocevar (coord.), Mendoza, abril 2010.

Paltridge, Brian; Starfield, Sue. Thesis and dissertation writing in a second language: a handbookfor supervisors. London: Routledge, 2007.

Parodi, Giovanni (Ed.) Alfabetización académica y profesional en el Siglo XXI: Leer y escribir desde las disciplinas. Santiago de Chile: Academia Chilena de la Lengua / Planeta, 2010.

----- Géneros académicos y Géneros Profesionales. Accesos discursivos para saber y hacer. Valparaíso: Ediciones Universitarias de Valparaíso. 2008a.

------ "La organización retórica del género Manual: ¿Una "colonia encadenada"?" Giovanni Parodi (Ed.). Géneros académicos y géneros profesionales: accesos discursivos para saber y hacer. Valparaíso: EUVSA, 2008b:169-198.

"El género Manual y su organización retórica en cuatro disciplinas científicas: Entre la abstracción y la concreción". Giovanni Parodi (Ed.). Géneros académicos y géneros profesionales: accesos discursivos para saber y hacer. Valparaíso: EUVSA. 2008c:199-218.

"El discurso especializado escrito en el ámbito universitario y profesional: constitución de un corpus de estudio". Revista Signos, 40 (63). 2007:147-178.

"Textos de especialidad y comunidades discursivas técnico-profesionales: Una aproximación a los manuales técnicos". Giovanni. Parodi (Ed.). Discurso especializado e instituciones formadoras. Valparaíso: EUVSA. 2005:61-80.

Sabaj Meruane, Omar; Toro Trengove, Paulina; Fuentes Cortés, Miguel. "Construcción de un modelo de movidas retóricas para el análisis de artículos de investigación del español". Onomázein, № 24. 2011:245-271.

Samraj, Betty. "A discourse analysis of master's theses across disciplines with a focus on introductions". Journal of English for Academic Purposes, 7. 2008:55-67.

Swales, John. Genre analysis: research genres. Explorations and applications. Cambridge: Cambridge University Press. 2004.

------ Genre analysis: English in academic and research settings. Cambridge: University of Cambridge. 1990. 
----- Aspects of article introductions. Birmingham, UK: The University of Aston, Language Studies Unit. 1981.

Tapia, Mónica; Burdiles, Gina; Arancibia, Beatriz. "Aplicación de una pauta diseñada para evaluar informes académicos universitarios". Revista Signos. 36 (54). 2003:249-257.

Tapia Ladino, Mónica; Burdiles, Gina. "Una caracterización del género informe escrito por estudiantes universitarios". Letras. Vol 51. № 79. 2009:17-49.

Tapia Ladino, Mónica; Marinkovich, Juanita. "Representaciones sociales sobre la escritura de la tesis en la formación académica inicial en el área de las Ciencias del Mar". Onomázein, № 24. 2011:273-297.

Venegas, René. "Clasificación de textos académicos en función de su contenido léxicosemántico". Revista Signos. vol.40, Nº3. 2007:239-271. 